OPEN ACCESS

Edited by:

Matjaž Perc

University of Maribor, Slovenia

Reviewed by:

Tai Sing Lee,

Carnegie Mellon University,

United States

Laurent U. Perrinet,

UMR7289 Institut de Neurosciences

de la Timone (INT), France

${ }^{*}$ Correspondence:

Zonglei Zhen

zhenzonglei@bnu.edu.cn

Jia Liu

liujiaTHU@tsinghua.edu.cn

Received: 30 June 2020 Accepted: 17 November 2020

Published: 09 December 2020

Citation:

Liu X, Zhen Z and Liu J (2020) Hierarchical Sparse Coding of Objects

in Deep Convolutional Neural

Networks.

Front. Comput. Neurosci. 14:578158

doi: 10.3389/fncom.2020.578158

\section{Hierarchical Sparse Coding of Objects in Deep Convolutional Neural Networks}

\author{
Xingyu Liu ${ }^{1}$, Zonglei Zhen ${ }^{1 *}$ and Jia Liu ${ }^{2 *}$ \\ ${ }^{1}$ Beijing Key Laboratory of Applied Experimental Psychology, Faculty of Psychology, Beijing Normal University, Beijing, China, \\ ${ }^{2}$ Department of Psychology \& Tsinghua Laboratory of Brain and Intelligence, Tsinghua University, Beijing, China
}

Recently, deep convolutional neural networks (DCNNs) have attained human-level performances on challenging object recognition tasks owing to their complex internal representation. However, it remains unclear how objects are represented in DCNNs with an overwhelming number of features and non-linear operations. In parallel, the same question has been extensively studied in primates' brain, and three types of coding schemes have been found: one object is coded by the entire neuronal population (distributed coding), or by one single neuron (local coding), or by a subset of neuronal population (sparse coding). Here we asked whether DCNNs adopted any of these coding schemes to represent objects. Specifically, we used the population sparseness index, which is widely-used in neurophysiological studies on primates' brain, to characterize the degree of sparseness at each layer in representative DCNNs pretrained for object categorization. We found that the sparse coding scheme was adopted at all layers of the DCNNs, and the degree of sparseness increased along the hierarchy. That is, the coding scheme shifted from distributed-like coding at lower layers to local-like coding at higher layers. Further, the degree of sparseness was positively correlated with DCNNs' performance in object categorization, suggesting that the coding scheme was related to behavioral performance. Finally, with the lesion approach, we demonstrated that both external learning experiences and built-in gating operations were necessary to construct such a hierarchical coding scheme. In sum, our study provides direct evidence that DCNNs adopted a hierarchically-evolved sparse coding scheme as the biological brain does, suggesting the possibility of an implementation-independent principle underling object recognition.

Keywords: deep convolutional neural network, sparse coding, coding scheme, object recognition, object representation, hierarchy

\section{INTRODUCTION}

One spectacular achievement of human vision is that we can accurately recognize objects at a fraction of a second in the complex visual world (Thorpe et al., 1996). In recent years, deep convolutional neural networks (DCNNs) have achieved human-level performances in object recognition tasks (He et al., 2015; Simonyan and Zisserman, 2015; Szegedy et al., 2015). The success is primarily credited to the architecture that generic DCNNs compose of a stack of convolutional layers and fully-connected layers, each of which has multiple units with different filters (i.e., 
"neurons" in DCNNs), similar to the hierarchical organization of primates' ventral visual stream. With such hierarchical architecture and supervised learning on a large number of object exemplars, DCNNs are thought to construct complex internal representations for external objects. However, little is known about how exactly objects are represented in DCNNs.

This question has already puzzled neuroscientists for a long time. To understand how primates' visual system encodes the external world, three types of coding schemes are proposed to describe how neurons are integrated together to represent an object. At one extreme is distributed coding, by which the whole neuronal population is involved, whereas at the other extreme is local coding, by which one neuron is designated to represent one object. The distributed coding scheme is superior in large coding capacity, easy generalization, and high robustness, while the local coding scheme is good at information compression, energy conservation and better interpretability. In between lies the sparse coding that different subsets of neurons in the population participate in coding different objects. As a trade-off, sparse coding possesses advantages of both local coding and distrusted coding (Barlow, 1972; Thorpe, 1989; Berkes et al., 2009; Rolls, 2017; Thomas and French, 2017; Beyeler et al., 2019). Neurophysiological studies have revealed that the sparse coding scheme is adopted in some areas in primate visual cortex for object recognition (Olshausen and Field, 1996; Lehky et al., 2011; Barth and Poulet, 2012; Rolls, 2017).

Following the studies on biological intelligent systems, several pioneer studies started to characterize DCNNs' representation with coding scheme (Szegedy et al., 2013; Agrawal et al., 2014; Li et al., 2016; Wang et al., 2016; Morcos et al., 2018; Casper et al., 2019; Parde et al., 2020). Studies using the ablation approach show that the processing of objects usually requires the participation of multiple units, but only $10-15 \%$ of units in a layer are actually needed to achieve $90 \%$ of the full performance (Agrawal et al., 2014). Even when half of the units in all layers are ablated, the performance does not decrease significantly with the accuracy above $90 \%$ of the full performance (Morcos et al., 2018). Further studies quantify the number of non-zero units in response to objects and report a trend of decrease in the number of non-zero units along the hierarchy of DCNNs (Agrawal et al., 2014). These preliminary results suggest that DCNNs may adopt the sparse coding scheme, which likely evolves along hierarchy.

Here, we adopted a prevalent metric in neurophysiological studies on primates' brain, population sparseness index (PSI, Rolls and Tovee, 1995; Vinje and Gallant, 2000), to quantify the population sparseness along the hierarchy of two representative DCNNs, AlexNet (Krizhevsky, 2014) and VGG11 (Simonyan and Zisserman, 2015). Specifically, we first systematically evaluated the layer-wise sparseness in representing objects. Then, we characterized the functionality of sparseness by examining the relationship between sparseness and behavioral performance in each layer. Finally, we explored factors that may influence the coding scheme.

\section{MATERIALS AND METHODS}

\section{Visual Images Datasets ImageNet Dataset}

The dataset from ImageNet Large Scale Visual Recognition Challenge 2012 (ILSVRC2012) (Russakovsky et al., 2015) contains 1,000 categories that are organized according to the hierarchy of WordNet (Miller, 1995). The 1,000 object categories consist of both internal nodes and leaf nodes of WordNet, but do not overlap with each other. The dataset contains 1.2 million images for model training, 50,000 images for model validation and 100,000 images for model test. In the present study, only the validation dataset (i.e., 1,000 categories $\times 50$ images) was used to evaluate the coding scheme of DCNNs.

\section{Caltech256 Dataset}

The Caltech256 dataset consists of 30,607 images from 256 object categories with a minimum number of 80 images per category (Griffin et al., 2007). In the present study, 80 images per category were randomly chosen from the original dataset.

\section{DCNNs and Activation Extraction}

The well-known AlexNet and VGG11 that are pretrained for object classification were selected to explore the coding scheme of DCNNs. Besides the two pretrained models, corresponding weight-permuted models and ReLU-deactivated models were also examined to investigate the factors that may influence the coding scheme observed in the pretrained models.

\section{Pretrained Models}

AlexNet and VGG11 are pretrained on ILSVRC2012 dataset and were downloaded from PyTorch model Zoo ${ }^{1}$. Both DCNNs are purely feedforward: the input to each layer consists solely of the output from the previous layer. The AlexNet consists of 5 convolutional layers (Conv1 through Conv5) that contain a set of feature maps with linear spatial filters, and 3 fullyconnected layers (FC1 through FC3). In between, a max (x, 0) rectifying non-linear unit (ReLU) is applied to all units after each convolutional and FC layer. In some convolutional layers, ReLU is followed by another max-pooling sublayer. VGG11 is similar to AlexNet in architecture except for two primary differences. First, VGG11 uses smaller receptive fields $(3 \times 3$ with a stride of 1$)$ than AlexNet $(11 \times 11$ with a stride of 4$)$. Second, VGG11 has more layers (8 convolutional layers) than AlexNet. When we refer to Conv\#, we mean the outputs from the ReLU sublayer in the \#th convolutional layer. Similarly, FC\# means the outputs from the \#th FC layer after ReLU. The DNNBrain toolbox ${ }^{2}$ was used to extract the DCNN activation (Chen et al., 2020). For each unit (or channel), the activation map was averaged to produce a unit-wise (or channel-wise) activation for each exemplar, and the activation of the unit to an object category was then derived by averaging the unit-wise responses from all exemplars of the category.

\footnotetext{
${ }^{1}$ https://pytorch.org/

${ }^{2}$ https://github.com/BNUCNL/dnnbrain/
} 
Weight-Permuted Models and Bias-Permuted Models The weight-permuted models were derived by permuting weights of the pretrained models within each layer. That is, the structures of the original networks and the weight distribution of each layer were preserved while the exact feature filters obtained from the learning of the supervised task were disrupted. Weights in a given layer can be decomposed as channel $\mathrm{x}$ kernel, in which kernels are 3-D tensors (i.e., input channel $\mathrm{x}$ height $\mathrm{x}$ width). Three kinds of permutation strategies with various scales were performed: weights were permutated across all channels and kernels, across channels with all kernels intact, and across kernels with channel orders unaltered. The bias-permuted models were obtained by permuting biases in each layer with all weights and the network structure remaining unchanged.

\section{ReLU-Deactivated Models}

The ReLU-deactivated model was the same as the pretrained models with only ReLU being silenced in all layers by replacing it with an identity mapping. The ReLU-deactivated model disabled the non-linear operation after the feature extraction but still retained the same network architectures and the learned feature filters.

\section{Population Sparseness Index}

The PSI was calculated for each layer of DCNNs to quantify the peakedness of the distribution of population responses elicited by an object category, which is equivalent to the fraction of the units in the population that participated in coding objects in the case of binary responses (Vinje and Gallant, 2000).

$$
\text { PSI }=\frac{1-a}{1-\frac{1}{N_{u}}}, \text { where } a=\frac{\left(\left(\sum r_{u}\right) / N_{u}\right)^{2}}{\sum\left(r_{u}^{2} / N_{u}\right)},
$$

where $r_{u}$ is the unit-wise activation of a unit $u$ from a target layer in response to an object category, and $N_{u}$ is the number of units in that layer. The unit-wise activation was z-scored across all categories for each unit, and then normalized across all units into a range from 0 to 1 to rescale the negative values to nonnegative as required by the definition of PSI. Values of PSI near 0 indicate low sparseness that all units respond equally to the object category, and values near 1 indicate high sparseness that only a few units respond to the category.

\section{Relationship Between Population Sparseness and Classification Performance}

The relationship between sparseness and classification performance was first explored using correlation analyses. The Caltech256 classification task was used to estimate the classification performance of AlexNet and VGG11 on each category. Specifically, a logistic regression model was constructed using activation patterns from FC2 as features to perform a 256-class object classification. A 2-fold cross-validation procedure was used to evaluate the classification performance. Then, Pearson correlation coefficients between the PSI and the classification performance were calculated across all categories for each layer, respectively. Finally, to reveal how the sparse coding from different layers contribute to the classification performance, a stepwise multiple regression was conducted with the classification performance of each category as dependent variables and the PSI of the corresponding category from all layers as independent variables. The regressions were conducted for Conv layers and FC layers separately.

\section{RESULTS}

The coding scheme for object categorization in DCNN was characterized layer by layer in the pretrained AlexNet and VGG11 using PSI. The PSI was first evaluated on the ImageNet validation dataset, with the same categories on which these two DCNNs were trained. Similar findings were revealed in the two DCNNs. First, the values of the PSI were low for all object categories in all layers in general (median $<0.4$ ), with the maximum values no larger than 0.6 (Figure 1), suggesting that the sparse coding scheme was broadly adopted in all layers of the DCNNs to represent objects. Second, in each layer, the PSI of all categories exhibited a broad distribution (ranges $>0.2$ ), indicating great individual differences in sparseness among object categories. However, despite the large amount of intercategory differences, the median PSI of each layer showed a trend of increase along the hierarchy in both Conv and FC layers, respectively (AlexNet: Kendall's tau $=0.40, p<$ 0.001; VGG11: Kendall's tau $=0.36, p<0.001$ ). A similar result was found with the absolute value of activation before computing the PSI (AlexNet: Kendall's tau $=-0.44, p<0.001$.; VGG11: Kendall's tau $=-0.52, p<0.001)$. Corroborative results were also observed by fitting the activation distribution of the neuron population with Norm distribution and Weibull functions (Supplementary Figure 1). Note that the increase in sparseness was not strictly monotonic, as the PSI of the first layer was slightly higher than the adjacent ones. More interestingly, although AlexNet and VGG11 have different numbers of Conv layers, the major increase occurred at the last Conv layer. Similar results have also been found in DCNNs (i.e., ResNet152 and GoogLeNet) whose architectures are significantly different from AlexNet and VGG11, suggesting that the hierarchical sparse coding scheme may be a general coding strategy in DCNNs (Supplementary Figure 2).

We replicated this finding with a new dataset, Caltech256, that is dissimilar to the ImageNet in object categories and is thus not in the training dataset. We found a similar pattern of the increase in sparseness along the hierarchy (AlexNet: Kendall's tau $=0.35, p<0.001 ;$ VGG11: Kendall's tau $=0.25, p<$ 0.001; Supplementary Figure 3), suggesting that the increase in sparseness did not result from image dataset. Taken together, the hierarchically-increased sparseness suggested that there was a systematic shift from the distributed-like coding scheme in low layers to the local-like coding scheme in high layers.

Next, we examined the functionality of the sparse coding scheme observed in the DCNNs. To address this question, we tested the association between the population sparseness and the behavioral performance by performing correlation analyses within each layer of the DCNNs. In AlexNet, significant 

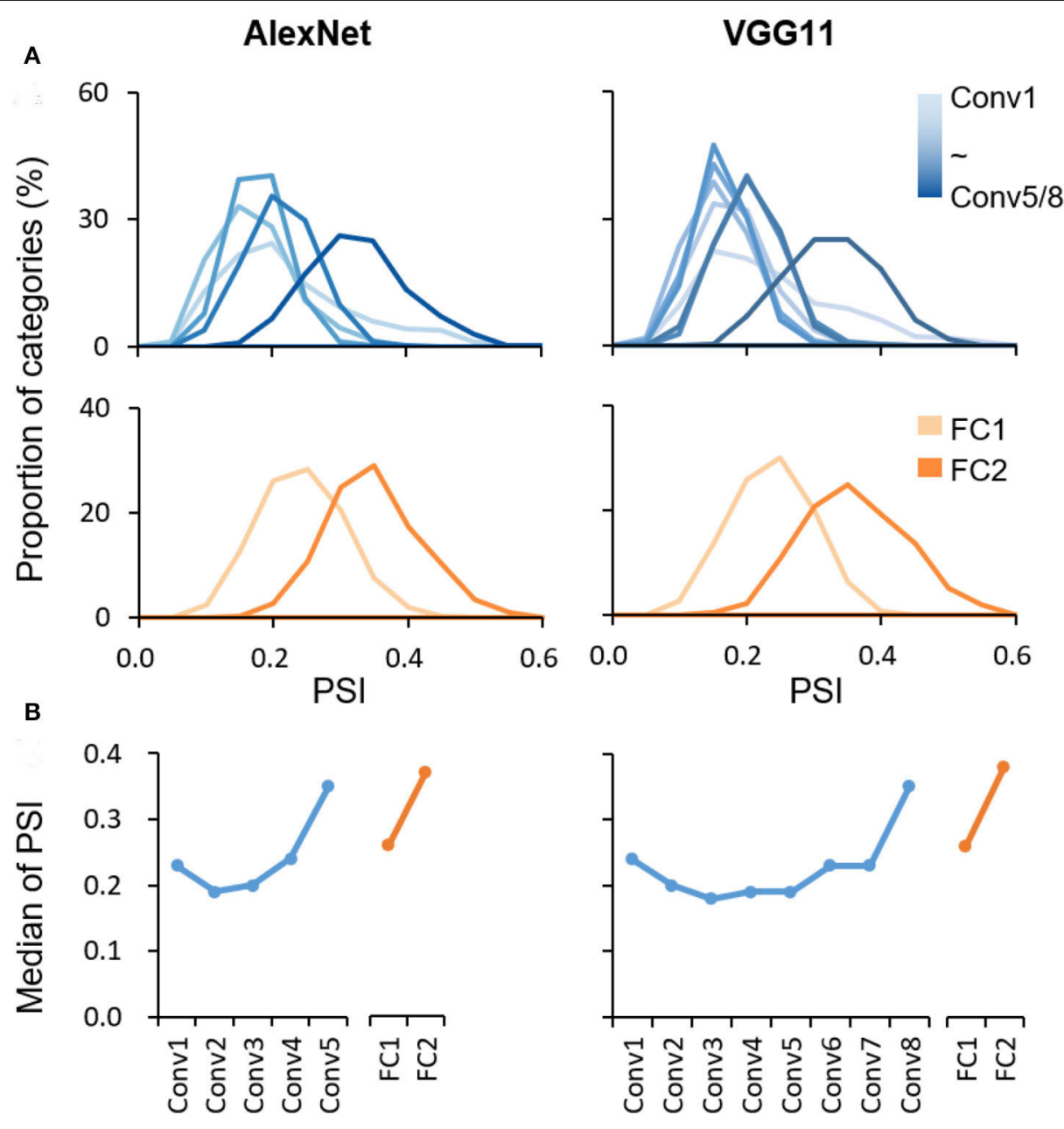

FIGURE 1 | Hierarchically sparse coding for object categories in DCNNs. (A) Layer-wise PSI distribution for object categories in DCNNs. The sparseness was evaluated using the PSI for each object category from the ImageNet dataset (1,000 categories) in each layer separately. The distribution of PSI right-shifted along hierarchy in general. $X$ axis: the degree of sparseness, with higher PSI indicating a higher degree of sparseness; $Y$ axis: the proportion of categories with a corresponding PSI value. (B) Median of PSI for each layer. In general, the median of PSI increased along hierarchy in Conv and FC layers, respectively. $X$ axis: the name of layers along hierarchy; $\mathrm{Y}$ axis: the median of PSI.

correlations were found starting from Conv4 and beyond $[r s$ (254) $>0.19$, ps < 0.001, Bonferroni corrected; Figure 2A]. This result suggested that the degree of sparseness in coding object categories was predictive of performance accuracy. That is, the sparser an object category was represented, the better it was recognized and classified. Importantly, the correlation coefficients also increased along hierarchy (Kendall's tau $=0.90$, $p=0.003)$, with the highest correlation coefficient observed at Conv5 (0.43) and FC2 (0.69), respectively (Figure 2A). This trend suggests a closer relationship between the population sparseness and the behavioral performance in higher layers. Indeed, with a stepwise multiple regression analysis in which PSI of all Conv/FC layers of certain categories were the independent variables and classification performance was the dependent variable, we confirmed that population sparseness was predictive of behavioral performance [Conv layers: $F_{(3,252)}=22.54, p$ $<0.001$, adjusted $R^{2}=0.2$; FC layers: $F_{(2,253)}=136.60, p$
$<0.001$, adjusted $R^{2}=0.52$ ]. Meanwhile, only PSI in higher layers starting from Conv3 remained in the regression models, further confirming that the coding scheme as a characteristic of representation became more essential with the increasing hierarchical level. Similar results were also found in VGG11 (Figure 2B), suggesting that the association between sparseness and performance may be universal in DCNNs.

Finally, we explored the factors that may affect the formation of such a hierarchical coding scheme in the DCNNs. The DCNNs consist of two subprocesses at the core of each layer (Figure 3A): one is the feature extraction process whose weights and biases are dynamically adjusted during learning, and the other is a gating process with a fixed non-linear function (i.e., ReLU) that silences units with negative activities. To examine whether the hierarchically-increased sparseness was constructed through learning, we measured the population sparseness of DCNNs with either the learned weights or biases randomly 


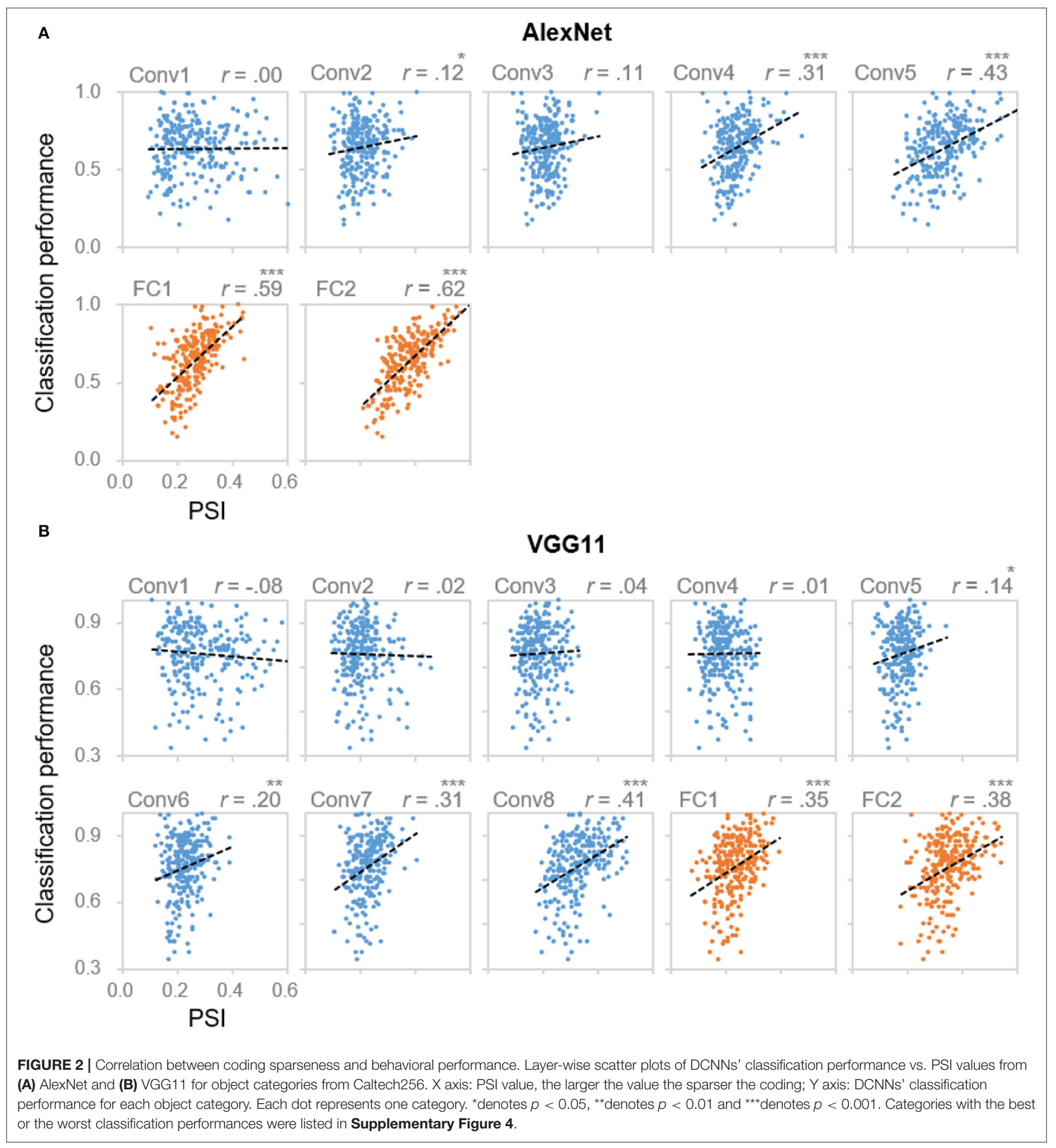

permuted. In the weight-permuted models where the weights were layer-wise permuted across all channels and kernels of the pretrained networks, we found that the degree of sparseness instead decreased along hierarchy (AlexNet: Kendall's tau = $-0.53, p<0.001$; VGG11: Kendall's tau $=-0.82, p<0.001$; Figure 3B), which was contradictory to the finding of the undisrupted one (Figure 1). This result was replicated when the weight permutation was performed across channels or kernels separately (AlexNet and VGG11: Kendall's taus <-0.53, ps $<0.001)$. Meanwhile, the population sparseness of the biaspermuted models in which all weights remained intact were also evaluated. We found that there was no increase in sparseness along hierarchy (AlexNet: Kendall's tau $=0.10, p=0.22$; VGG11: Kendall's tau $=-0.15, p=0.03$; Figure 3D). In addition, when 


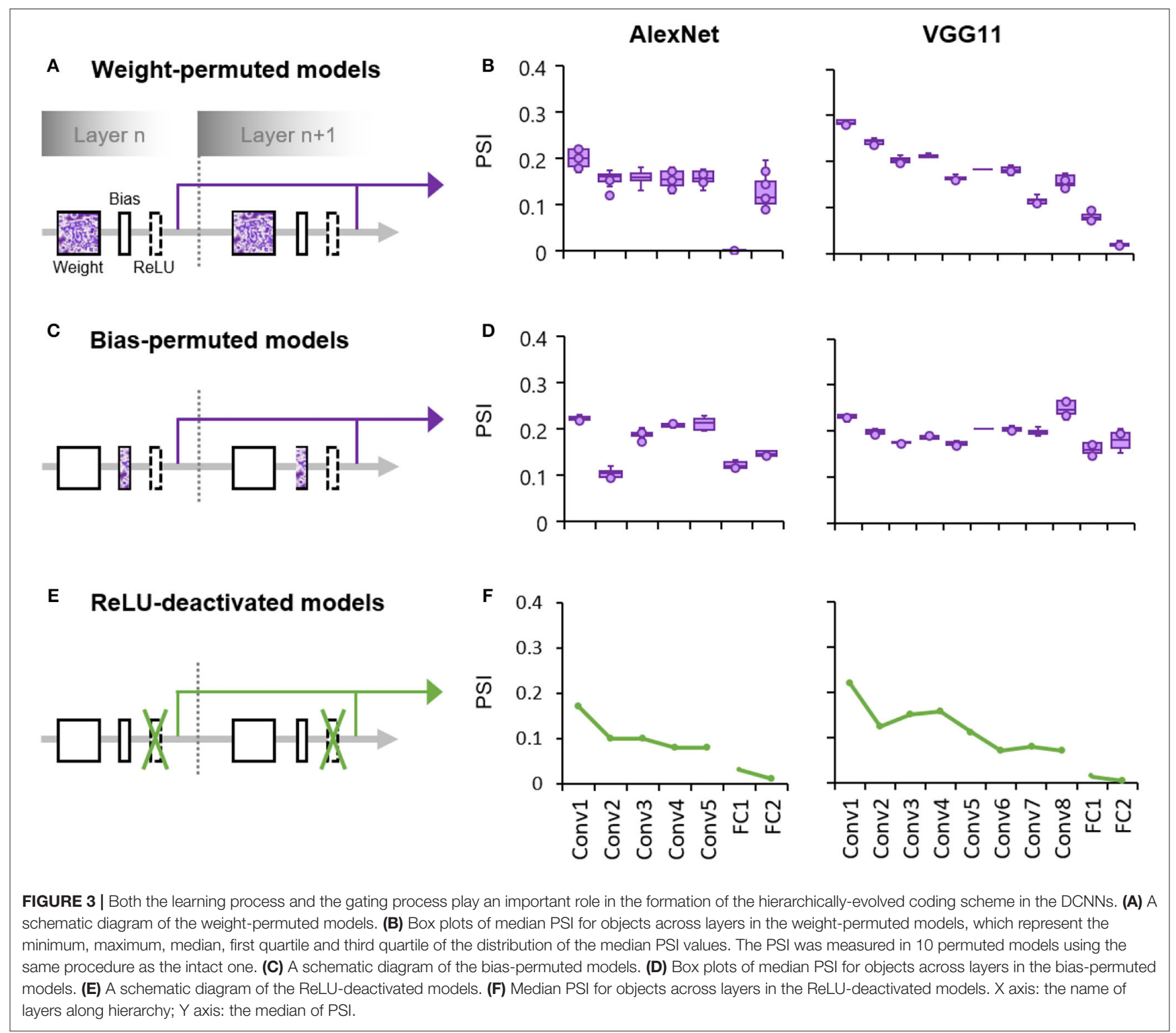

the ReLU sublayers were deactivated with the feature extraction sublayers intact (Figure 3E), we also observed a decreasing tendency of sparseness along the hierarchy (AlexNet: Kendall's tau $=-0.21, p<0.001 ;$ VGG11: Kendall's tau $=-0.32, p$ $<0.001$, Figure 3F), again in contrast to the AlexNet with functioning ReLU (Figure 1). Similar results were also found in VGG11, suggesting a general effect of learning and gating on the formation of the hierarchically-evolved coding scheme in DCNN.

\section{DISCUSSION}

In the present study, we systematically characterized the coding scheme in representing object categories at each layer of two typical DCNNs, AlexNet, and VGG11. We found that objects were in general sparsely encoded in the DCNNs, and the degree of sparseness increased along the hierarchy. Importantly, the hierarchically-evolved sparseness was able to predict the classification performance of the DCNNs, revealing the functionality of the sparse coding. Finally, lesion analyses of the weight-permuted models, the bias-permuted models, and the ReLU-deactivated models suggest that the learning experience and the built-in gating operation account for the hierarchically sparse coding scheme in the DCNNs. In short, our study provided one of the empirical evidence illustrating how object categories were represented in DCNNs for object recognition.

The finding that the degree of sparseness increased along the hierarchy in DCNNs is consistent with previous studies on DCNNs (Szegedy et al., 2013; Agrawal et al., 2014; Tripp, 2016; Wang et al., 2016; Morcos et al., 2018; Casper et al., 2019; Parde et al., 2020). Our study further extended these previous studies by conducting a layer-wise analysis throughout all hierarchical levels and calculating the degree of sparseness based on responses of the entire population of units ("neurons" in DCNN). Besides, our study tested two datasets of more than 
1,000 object categories, and thus provided more comprehensive coverage of the object space. Finally, we also examined the functionality of sparse coding by showing that the sparser an object category was encoded, the higher accuracy of the object category was correctly recognized.

The fact that the hierarchically-increased coding sparseness coincides with a hierarchically-higher behavioral relevance in DCNNs suggests it as an organizing principle of representing a myriad of objects efficiently. That is, at the lower level of vision, representations recruit a larger number of generic neurons to process myriad natural objects with high fidelity. At the higher level, objects are decomposed into abstract features in the object space; therefore, only a smaller but highly-specialized group of neurons are recruited to construct the representation. Critically, a higher degree of sparseness makes representations more interpretable, because only at higher layers the degree of sparseness was able to read out for behavioral performance. One possibility is that distributed coding adopts more neuronal crosstalk that is difficult for readout, whereas sparser coding contains fewer higher-order relations and hence require less amount of computation for object recognition and memory storage/retrieval (Field, 1994; Froudarakis et al., 2014; Beyeler et al., 2019). That is, distributed coding is better at adapting and generalizing the variance across stimulus exemplars; sparse coding serves to explicit interpretation for goal-directed invariance (Földiák, 2009; Babadi and Sompolinsky, 2014; King et al., 2019). Taken together, the evolution of sparseness along the hierarchy likely mirrored the stages of objects being processed and the transformation of representation from stimulus-fidelity to goal-fidelity.

Interestingly, the sparseness was not accumulated gradually layer by layer. Instead, the sparseness was the highest at the last convolutional layer (i.e., Conv5 in AlexNet and Conv8 in VGG11) and fully-connected layer (i.e., FC2 in AlexNet and VGG11), much higher than that of their preceding ones regardless of the total number of layers in the DCNNs. This observation suggests a mechanism that the degree of sparseness dramatically increases at transitional layers either to the next processing stage (from Conv layers to FC layers) or to the generation of behavioral performance (from FC layers to the output layer). Further studies are needed to explore the functionality of the dramatic increase in sparseness. Note that the finding that the increase of sparseness was observed in two structurally-similar DCNNs (i.e., AlexNet and VGG11), and therefore it may not be applicable to other DCNNs.

As an intelligent system, DCNNs are products of the predesigned architecture by nature and learned features by nurture. Our lesion study revealed that both architecture and learning were critical for the formation of the hierarchically sparse coding scheme. As for the innate architecture, a critical built-in function is the non-linear gating sublayer, ReLU, that silences neurons with negative activity (Glorot et al., 2011; LeCun et al., 2015). Obviously, the gating function is bound to increase the sparseness of coding because it removes weak or irrelevant activations and thus leads objects to be represented by a smaller number of units. Our study confirmed this intuition by showing the disruption of hierarchically-increased sparseness when the gating function being disabled. Besides the commonly used gating operation ReLU, recently more approaches have been developed to directly serve the same purpose of sparsification (Liu et al., 2015; Kepner et al., 2018). On the other hand, the gating function was not sufficient for a proper sparse coding scheme, because after randomly permuting the weights of the learned filters in the feature sublayers, the sparseness was no longer properly constructed either. Further, the dependence of both external learning experiences and built-in non-linear operations implies that the sparse coding scheme may be also adopted in biological brains, because the gating function is the fundamental function of neurons (Lucas, 1909; Adrian, 1914) and the deprivation of visual experiences leads to deficits in a variety of visual functions (Wiesel and Hubel, 1963; Fine et al., 2003; Duffy and Livingstone, 2005). In short, the current study provides direct empirical evidence on the functionality and formation of hierarchy-dependent coding sparseness in DCNNs; However, the exact computational mechanisms underlying the evolution of sparse coding along hierarchy are needed for future work to unravel it.

Our findings with biologically-inspired DCNNs also lend insight into coding schemes in biological systems. Because the number of object categories, neurons, and sampling sites are largely limited by neurophysiological techniques, availability of subjects and ethical issues, it is difficult to characterize population sparseness along the visual pathway (Baddeley et al., 1997; Vinje and Gallant, 2000; Tolhurst et al., 2009). Several studies measured the population sparseness on certain single regions in mouse, ferret or macaque brain (Berkes et al., 2009; Froudarakis et al., 2014; Tang et al., 2018), but with diverse experimental setups, the evolution of population sparseness across brain regions is unclear. Lenky et al. did record both a group of V1 and the Inferotemporal neurons and found that the population sparseness increased from the V1 to Inferotemporal cortex (Lehky et al., 2005, 2011). In contrast, DCNNs can be used to examine not only coding schemes of a large number of objects ( $>1,000$ object categories in our study) but also the degree of the sparseness of all units in all layers; therefore, DCNNs may serve as a quick-and-dirty model to pry open how visual information is represented in biological systems.

In sum, our study on the coding scheme of object categories in DCNNs invites future studies to understand how in DCNN objects are recognized accurately in particular, and how intelligence emerges under the interaction of internal architecture and external learning experiences in general. On one hand, approaches and findings from neurophysiological and fMRI studies help to transpire the black-box of DCNNs and enlighten the design of more effective DCNNs. For example, our study suggests new algorithms for better performance by increasing sparseness effectively possibly through learning or gating function built in the network. On the other hand, in contrast to the fact that neurophysiological studies on nonhuman primates and fMRI studies on human are limited either by the coverage of brain areas or by the spatial resolution, both architecture and units' activation in DCNNs are transparent. Therefore, DCNNs likely provides a perfect model to pry open mechanisms of object recognition at both microand macro-levels, which helps to understand how biological intelligent systems work. 


\section{DATA AVAILABILITY STATEMENT}

Datasets analyzed in the present article were from two public datasets: (1) ImageNet: http://www.image-net.org/; (2) Caltech256: http://www.vision.caltech.edu/Image_Datasets/ Caltech256. All codes for activation extraction and analyses are available on https://github.com/xingyu-liu/coding_sparseness.

\section{AUTHOR CONTRIBUTIONS}

$\mathrm{XL}, \mathrm{ZZ}$, and JL conceived the study and wrote the manuscript. XL developed the code and performed the research. All authors contributed to the article and approved the submitted version.

\section{REFERENCES}

Adrian, E. D. (1914). The all-or-none principle in nerve. J. Physiol. 47, 460-474. doi: 10.1113/jphysiol.1914.sp001637

Agrawal, P., Girshick, R., and Malik, J. (2014). "Analyzing the performance of multilayer neural networks for object recognition," in Computer Vision - ECCV 2014, eds D. Fleet, T. Pajdla, B. Schiele, and T. Tuytelaars (Cham: Springer International Publishing), 329-344. doi: 10.1007/978-3-319-10584-0_22

Babadi, B., and Sompolinsky, H. (2014). Sparseness and expansion in sensory representations. Neuron 83, 1213-1226. doi: 10.1016/j.neuron.2014.07.035

Baddeley, R., Abbott, L. F., Booth, M. C. A., Sengpiel, F., Freeman, T., Wakeman, E. A., et al. (1997). Responses of neurons in primary and inferior temporal visual cortices to natural scenes. Proc. R. Soc. Lond. B 264, 1775-1783. doi: 10.1098/rspb.1997.0246

Barlow, H. B. (1972). Single units and sensation: a neuron doctrine for perceptual psychology? Perception 1, 371-394. doi: 10.1068/p010371

Barth, A. L., and Poulet, J. F. A. (2012). Experimental evidence for sparse firing in the neocortex. Trends Neurosci. 35, 345-355. doi: 10.1016/j.tins.2012.03.008

Berkes, P., White, B., and Fiser, J. (2009). "No evidence for active sparsification in the visual cortex," in Advances in Neural Information Processing Systems, eds Y. Bengio, D. Schuurmans, J. D. Lafferty, C. K. I. Williams, and A. Culotta (Red Hook, NY: Curran Associates, Inc.), 108-116. Available online at: http://papers.nips.cc/paper/3774-no-evidence-for-active-sparsification-inthe-visual-cortex.pdf (accessed November 18, 2019).

Beyeler, M., Rounds, E. L., Carlson, K. D., Dutt, N., and Krichmar, J. L. (2019). Neural correlates of sparse coding and dimensionality reduction. PLoS Comput. Biol. 15:e1006908. doi: 10.1371/journal.pcbi.1006908

Casper, S., Boix, X., D’Amario, V., Guo, L., Schrimpf, M., Vinken, K., et al. (2019). Removable and/or repeated units emerge in overparametrized deep neural networks. arXiv:1912.04783 [cs, stat]. Available online at: http://arxiv.org/abs/ 1912.04783 (accessed June 26, 2020).

Chen, X., Zhou, M., Gong, Z., Xu, W., Liu, X., Huang, T., et al. (2020). DNNBrain: A Unifying Toolbox for Mapping Deep Neural Networks and Brains. Front. Comput. Neurosci. 14:580632. doi: 10.3389/fncom.2020. 580632

Duffy, K. R., and Livingstone, M. S. (2005). Loss of neurofilament labeling in the primary visual cortex of monocularly deprived monkeys. Cerebral Cortex 15, 1146-1154. doi: 10.1093/cercor/bhh214

Field, D. J. (1994). What is the goal of sensory coding? Neural Comput. 6, 559-601. doi: 10.1162/neco.1994.6.4.559

Fine, I., Wade, A. R., Brewer, A. A., May, M. G., Goodman, D. F., Boynton, G. M., et al. (2003). Long-term deprivation affects visual perception and cortex. Nat. Neurosci. 6, 915-916. doi: 10.1038/nn1102

Földiák, P. (2009). Neural coding: non-local but explicit and conceptual. Curr. Biol. 19, R904-R906. doi: 10.1016/j.cub.2009.08.020

Froudarakis, E., Berens, P., Ecker, A. S., Cotton, R. J., Sinz, F. H., Yatsenko, D., et al. (2014). Population code in mouse V1 facilitates readout of natural scenes through increased sparseness. Nat. Neurosci. 17, 851-857. doi: 10.1038/ nn. 3707

\section{FUNDING}

This study was funded by the National Natural Science Foundation of China (Grant No. 31861143039 and 31771251), and the National Basic Research Program of China (Grant No. 2018YFC0810602), the National Key R\&D Program of China (Grant No. 2019YFA0709503).

\section{SUPPLEMENTARY MATERIAL}

The Supplementary Material for this article can be found online at: https://www.frontiersin.org/articles/10.3389/fncom. 2020.578158/full\#supplementary-material

Glorot, X., Bordes, A., and Bengio, Y. (2011). "Deep sparse rectifier neural networks," in International Conference on Artificial Intelligence and Statistics (Fort Lauderdale, FL), 315-323.

Griffin, G., Holub, A., and Perona, P. (2007). Caltech-256 Object Category Dataset. California Institute of Technology. Available online at: https://resolver.caltech. edu/CaltechAUTHORS:CNS-TR-2007-001

He, K., Zhang, X., Ren, S., and Sun, J. (2015). Delving deep into rectifiers: surpassing human-level performance on ImageNet classification. in 2015 IEEE International Conference on Computer Vision (ICCV) (Santiago), 1026-1034. doi: 10.1109/ICCV.2015.123

Kepner, J., Gadepally, V., Jananthan, H., Milechin, L., and Samsi, S. (2018). "Sparse deep neural network exact solutions," in 2018 IEEE High Performance extreme Computing Conference (HPEC) (Waltham, MA), 1-8. doi: 10.1109/HPEC.2018.8547742

King, M. L., Groen, I. I. A., Steel, A., Kravitz, D. J., and Baker, C. I. (2019) Similarity judgments and cortical visual responses reflect different properties of object and scene categories in naturalistic images. NeuroImage 197, 368-382. doi: 10.1016/j.neuroimage.2019.04.079

Krizhevsky, A. (2014). One weird trick for parallelizing convolutional neural networks. arXiv:1404.5997 [cs]. Available online at: http://arxiv.org/abs/1404. 5997 (accessed June 26, 2020).

LeCun, Y., Bengio, Y., and Hinton, G. (2015). Deep learning. Nature 521, 436-444 doi: $10.1038 /$ nature14539

Lehky, S. R., Kiani, R., Esteky, H., and Tanaka, K. (2011). Statistics of visual responses in primate inferotemporal cortex to object stimuli. J. Neurophysiol. 106, 1097-1117. doi: 10.1152/jn.00990.2010

Lehky, S. R., Sejnowski, T. J., and Desimone, R. (2005). Selectivity and sparseness in the responses of striate complex cells. Vis. Res. 45, 57-73. doi: 10.1016/j.visres.2004.07.021

Li, Y., Yosinski, J., Clune, J., Lipson, H., and Hopcroft, J. (2016). Convergent learning: do different neural networks learn the same representations? arXiv:1511.07543 [cs]. Available online at: http://arxiv.org/abs/1511.07543 (accessed March 11, 2020).

Liu, B., Wang, M., Foroosh, H., Tappen, M., and Penksy, M. (2015). "Sparse convolutional neural networks," in 2015 IEEE Conference on Computer Vision and Pattern Recognition (CVPR) (Boston, MA: IEEE), 806-814.

Lucas, K. (1909). The "all or none" contraction of the amphibian skeletal muscle fibre. J. Physiol. 38, 113-133. doi: 10.1113/jphysiol.1909.sp001298

Miller, G. A. (1995). WordNet: a lexical database for English. Commun. ACM 38, 39-41. doi: 10.1145/219717.219748

Morcos, A. S., Barrett, D. G. T., Rabinowitz, N. C., and Botvinick, M. (2018). On the importance of single directions for generalization. arXiv:1803.06959 [cs, stat]. Available online at: http://arxiv.org/abs/1803.06959 (accessed December 20, 2019).

Olshausen, B. A., and Field, D. J. (1996). Emergence of simple-cell receptive field properties by learning a sparse code for natural images. Nature 381, 607-609. doi: $10.1038 / 381607 \mathrm{a} 0$

Parde, C. J., Colón, Y. I., Hill, M. Q., Castillo, C. D., Dhar, P., and O’Toole, A. J. (2020). Single unit status in deep convolutional neural network codes for face 
identification: sparseness redefined. arXiv:2002.06274 [cs]. Available online at: http://arxiv.org/abs/2002.06274 (accessed March 19, 2020).

Rolls, E. T. (2017). Cortical coding. Lang. Cogn. Neurosci. 32, 316-329. doi: $10.1080 / 23273798.2016 .1203443$

Rolls, E. T., and Tovee, M. J. (1995). Sparseness of the neuronal representation of stimuli in the primate temporal visual cortex. J. Neurophysiol. 73, 713-726. doi: 10.1152/jn.1995.73.2.713

Russakovsky, O., Deng, J., Su, H., Krause, J., Satheesh, S., Ma, S., et al. (2015). ImageNet large scale visual recognition challenge. Int. J. Comput. Vis. 115, 211-252. doi: 10.1007/s11263-015-0816-y

Simonyan, K., and Zisserman, A. (2015). Very deep convolutional networks for large-scale image recognition. arXiv:1409.1556 [cs]. Available online at: http:// arxiv.org/abs/1409.1556 (accessed June 26, 2020).

Szegedy, C., Wei, L, Yangqing, J, Sermanet, P., Reed, S., Anguelov, D., et al. (2015). "Going deeper with convolutions," in 2015 IEEE Conference on Computer Vision and Pattern Recognition (CVPR) (Boston, MA), 1-9. doi: 10.1109/CVPR.2015.7298594

Szegedy, C., Zaremba, W., Sutskever, I., Bruna, J., Erhan, D., Goodfellow, I., et al. (2013). Intriguing properties of neural networks. arXiv:1312.6199 [cs]. Available online at: http://arxiv.org/abs/1312.6199 (accessed September 1, 2019).

Tang, S., Zhang, Y., Li, Z., Li, M., Liu, F., Jiang, H., et al. (2018). Large-scale twophoton imaging revealed super-sparse population codes in the V1 superficial layer of awake monkeys. eLife 7:e33370. doi: 10.7554/eLife.33370.015

Thomas, E., and French, R. (2017). Grandmother cells: much ado about nothing. Lang. Cogn. Neurosci. 32, 342-349. doi: 10.1080/23273798.2016.1235279

Thorpe, S. (1989). Local vs. distributed coding. Intellectica 8, 3-40. doi: 10.3406/intel.1989.873

Thorpe, S., Fize, D., and Marlot, C. (1996). Speed of processing in the human visual system. Nature $381,520-522$. doi: $10.1038 / 381520 a 0$
Tolhurst, D. J., Smyth, D., and Thompson, I. D. (2009). The sparseness of neuronal responses in ferret primary visual cortex. J. Neurosci. 29, 2355-2370. doi: 10.1523/JNEUROSCI.3869-08.2009

Tripp, B. (2016). Similarities and differences between stimulus tuning in the inferotemporal visual cortex and convolutional networks. arXiv:1612.06975 [qbio]. Available online at: http://arxiv.org/abs/1612.06975 (accessed March 19, 2020).

Vinje, W. E., and Gallant, J. L. (2000). Sparse coding and decorrelation in primary visual cortex during natural vision. Science 287, 1273-1276. doi: 10.1126/science.287.54 56.1273

Wang, J., Zhang, Z., Xie, C., Premachandran, V., and Yuille, A. (2016). Unsupervised learning of object semantic parts from internal states of CNNs by population encoding. arXiv:1511.06855 [cs]. Available online at: http://arxiv. org/abs/1511.06855 (accessed June 26, 2020).

Wiesel, T. N., and Hubel, D. H. (1963). Effects of visual deprivation on morphology and physiology of cells in the cat's lateral geniculate body. J. Neurophysiol. 26, 978-993. doi: 10.1152/jn.1963. 26.6.978

Conflict of Interest: The authors declare that the research was conducted in the absence of any commercial or financial relationships that could be construed as a potential conflict of interest.

Copyright $\odot 2020 \mathrm{Liu}$, Zhen and Liu. This is an open-access article distributed under the terms of the Creative Commons Attribution License (CC BY). The use, distribution or reproduction in other forums is permitted, provided the original author(s) and the copyright owner(s) are credited and that the original publication in this journal is cited, in accordance with accepted academic practice. No use, distribution or reproduction is permitted which does not comply with these terms. 